\title{
The Factors Affecting Long Term Tricuspid Regurgitation After Double Valve Replacement
}

\author{
REZAN AKSOY ${ }^{1}$, Deniz Cevirme ${ }^{1}$, Mehmet Dedemoğlu², Hakan Hancer ${ }^{3}$, Alev \\ Kilicgedik $^{3}$, and Murat Rabus ${ }^{4}$ \\ ${ }^{1}$ University of Health Sciences, Kosuyolu Heart Education and Research Hospital, Istanbul, \\ Turkey \\ ${ }^{2}$ University of Health Sciences, Umraniye Education and Research Hospital \\ ${ }^{3}$ University of Health Sciences, Kosuyolu Heart Education and Research Hospital \\ ${ }^{4}$ Affiliation not available
}

May 15, 2020

\begin{abstract}
Background: This study aims to determine the hemodynamic performance of double valve prostheses and its effects on long term outcomes of tricuspid regurgitation(TR) following double valve replacement (DVR). Methods: Between January 2014September 2017, all patients undergoing concomitant aortic and mitral valve replacement with or without concomitant tricuspid valve repair at the same center were reviewed. All patients were divided into three groups according to interventions tricuspid valve(TVI), that is no-tricuspid annuloplasty(TAP), tricuspid ring annuloplasty and tricuspid de-vega annuloplasty (TDVA).Results: The study included 179 patients, of whom $52 \%$ were women(with mean age $51.7 \pm 13.7$ years). Of 179 patients, 100 were in the no-TAP group, 39 were in the ring annuloplasty group and 40 were in the TDVA group. There was no statically significant difference among the groups with regard to potential complications, in-hospital mortality and the survival rates. The degree of TR decreased with TVI. The rates of moderate and severe TR were significantly lower in in the ring annuloplasty group $(\mathrm{P}=0.04$ and 0.004$)$. Female gender $(\mathrm{OR}=4.0,95 \% \mathrm{CI}: 1.0-15.5, \mathrm{p}=0.05)$ and preoperative $\mathrm{TR} \operatorname{degree}(\mathrm{OR}=2.9$, 95\% CI: 1.1-7.3, $\mathrm{p}=0.01)$ were an independent predictors for the development of postoperative severe TR. Conclusion: Although the degree of postoperative TR varies according to whether to perform a TVI, DVR can be safe due to low operative mortality independently from TVI. The rates of moderate and severe TR were significantly lower in the ring annuloplasty group and female gender was an independent risk factor of TR progression.
\end{abstract}

\section{Introduction}

Double valve replacement (DVR) is a more complex procedure than isolated aortic or mitral valve replacement (1). In patients with DVR, the presence of a mitral valve prosthesis may be related to adverse effect of aortic valve prostheses (2). Following left-sided valve surgery, left sided valve dysfunction with tricuspid regurgitation(TR) may occur owing to the fact that progression of the rheumatic process in native heart valves or prosthetic valve dysfunction(3). Recently, studies have shown that DVR was more strongly related to the late severe tricuspid regurgitation $(3,4)$. Functional tricuspid regurgitation (TR) is usually accompanied by mitral valve (MV) diseases, and the presence of a serious TR is associated with unfavorable prognosis followed by mitral valve surgery (5). Tricuspid valve annuloplasty (TVA) using annuloplasty bands or rings has more satisfactory outcomes of recurrent tricuspid regurgitation (TR) than suture annuloplasty(6-8).

It is of clinical significance to prevent late TR, since TR has negative impacts on long term morbidity and mortality, surgical intervention of TR following mitral valve surgery results in greatly increased morbidity 
and mortality (9-11). Elevated TR after DVR is commonly encountered (12,13), but its underlying causes remain to be refined.

This study aims to determine the hemodynamic performance of double valve prostheses and its effects on long term outcomes of TR following DVR.

\section{Materials and Methods}

Between January 2014 and September 2017, all patients undergoing concomitant aortic and mitral valve replacement, double valve replacement (DVR), with or without concomitant tricuspid valve repair at the same center were reviewed retrospectively. The inclusion criteria were only the cases who electively underwent DVR with no other concomitant cardiac intervention except tricuspid valve repair. The exclusion criteria were patients undergoing emergency surgery, mitral or aortic valve repair surgeries, concomitant cardiac surgeries, patients with unstable cardiac condition, patients who required care in the intensive care unit (ICU) before surgery and those with infective endocarditis. As a result, a total of 179 consecutive patients were included into the study. All patients were divided into three groups according to interventions tricuspid valve that is no-tricuspid annuloplasty (TAP), tricuspid ring annuloplasty and tricuspid de-vega annuloplasty (TDVA).

\section{Surgical procedure}

All surgeries were performed using aortic arterial and bi-caval venous cannulations for cardiopulmonary bypass (CPB). Myocardial protection with mild hypothermia was achieved by intermittent antegrade cardioplegia and /or retrograde cardioplegia. While the mechanical prosthetic valve was the most commonly used material as the artificial valve, biological prosthetic valve replacement was performed for patients with elderly or women considering pregnancy. After the completion of aortic and mitral valve replacement, in patients undergoing TDVA, tricuspid annuloplasty was performed using double row-pledgeted stitching through right atriotomy. Surgeon decided to technique of tricuspid intervention. When the ring annuloplasty was planned for tricuspid valve repair we used a 3D rigid tricuspid ring for all patients. After the operation, all patients were transferred to the cardiac intensive care unit for the postoperative management. During the postoperative period, warfarin was recommended as a lifelong oral anticoagulant treatment to the all patients with a mechanical prosthetic valve.

\section{Evaluation of tricuspid regurgitation}

All patients had an echocardiographic examination before and after the operation. The echocardiographic findings were evaluated by echocardiographic cardiologists. The degree of TR was evaluated using the apical four-chamber view on postoperative period. The severity of tricuspid regurgitation (TR) was defined as 0 for no-TR, as 1 for trivial TR, as 2 for mild TR, as 3 for moderate TR and as 4 for severe TR. Surgeons performed to tricuspid valve intervention in TR patients who has moderate and severe TR or dilated annulus $\left(>\_40 \mathrm{~mm}\right)$.

\section{Outcomes and follow-up}

All patients were followed-up by physical and echocardiographic examination in the postoperative period. The valve functions and the presence of TR regurgitation were evaluated. The other outcome of interest was to compare the groups in terms of postoperative outcomes and long-term survival rates. We included last postoperative echocardiographic results of patients.

\section{Statistical analyses}

Statistical analyses were performed by using SPSS (Statistical Package for the Social Sciences) software. Parametric continuous data were expressed as mean and standard deviation, while non-parametric continuous data as median and interquartile ranges. Besides the categorical data were defined as frequency and percentage. For the independent group comparisons, one-way analysis of variance (ANOVA) was used for parametric continuous data, while Kruskal Wallis test for non-parametric continuous data and Pearson ChiSquare test for categoric data. 'Post Hoc tests' were utilized for checking the analyses. For the dependent 
group comparisons, Paired sample T test was used for parametric continuous data, while Wilcoxon test for non-parametric continuous data. To determine independent predictors for dependent variable, multivariate logistic regression analysis was used. The appropriateness of model was tested using Backward: Wald approach. Long-term survival curves were computed using Kaplan-Meier method and Log-rank test was used to compare survival rates of the groups. For all statistical analyses, p value less than 0.05 was defined as a statistical significance.

\section{Results}

The study included 179 patients, of whom $52 \%$ were women (with mean age $51.7 \pm 13.7$ years). Of 179 patients, 100 were in the no-TAP group, 39 were in the ring annuloplasty group and 40 were in the TDVA group. The characteristics of the groups are shown in Table 1 . The demographic data and findings were mostly similar among the groups. The only parameter of atrial fibrillation (AF) was significantly lower in no-TAP group $(\mathrm{p}=0.001)$. Table 2 shows preoperative echocardiographic findings.

Table 1: Preoperative Patient Characteristics

Table 2: Preoperative Echocardiographic Findings

No-TAP group had significantly lower levels of maximum mitral gradient, PAP and degree of TR compared with the other two groups $(\mathrm{P}=0.04,<0.001$ and $<0.001$ respectively). Additionally, No-TAP group had significantly shorter tricuspid valve diameter and left atrial diameter (LAD) than the other groups (P $<0.001$ and 0.01 ). The other parameters were similar among the groups. In terms of operative findings, CPB duration increased with tricuspid intervention. In particular, CPB duration was significantly longer in ring annuloplasty group $(\mathrm{P}=0.001)$. Looking at the postoperative outcomes (Table 3 ), there was no statically significant difference among the groups with regard to potential complications and in-hospital mortality. On the other hand, the durations of mechanical ventilation and ICU stay were significantly shorter in no-TAP group $(\mathrm{P}=0.02$ and 0.001$)$. This may possibly be related to the fact that no-TAP had shorter CPB time.

Table 3: Postoperative Outcomes

Table 4 shows postoperative echocardiographic findings. The degree of TR decreased with tricuspid intervention. Furthermore, the ring annuloplasty group had significantly lower TR degree than the other two groups $(\mathrm{P}=0.01)$. Meanwhile, the rates of moderate and severe $\mathrm{TR}$ were significantly lower in in the ring annuloplasty group $(\mathrm{P}=0.04$ and 0.004$) .91$ of patients were rheumatic valvular pathologies and 88 of patients were degenerative valvular pathologies. Following DVR, degree of postoperative TR was not significant difference according to ethology $(\mathrm{p}=0.05)$. However female patients have more rheumatic valvular pathologies than male patients (male patients, n: 37(\%43), female patients; n: 54(\%58), p: 0.04).

Table 4: Postoperative Echocardiographic Findings

On the other hand, LAD was shown to be significantly larger in the TDVA group $(\mathrm{P}=0.04)$. The possible reason may be related to the fact that in the TDVA group had a large LAD before the surgery and there was no significant regression on LAD after the surgery.

Looking at the comparison of pre- and postoperative echocardiographic findings in terms of groups it was observed that all echocardiographic parameters mostly regressed for all groups. However, the degree of TR did not decrease significantly in the no-TAP group $(\mathrm{P}=0.1)$. In contrast, the degree of $\mathrm{TR}$ decreased significantly both in the ring annuloplasty and in the TDVA groups $(\mathrm{P}<0.001$ for both). BMI, degree of preoperative TR and diameter of tricuspid annulus were not significantly difference according to gender.

As a result of multivariate analysis, no-TAP during surgery was associated with severe TR $(\mathrm{OR}=27.5$, 95\% CI: 3.9-193.2, $\mathrm{p}=0.01)$. In addition to this, female gender $(\mathrm{OR}=4.0,95 \% \mathrm{CI}: 1.0-15.5, \mathrm{p}=0.05)$ and preoperative $\mathrm{TR}$ degree $(\mathrm{OR}=2.9,95 \% \mathrm{CI}: 1.1-7.3, \mathrm{p}=0.01)$ were the other independent predictors for the development of postoperative severe TR.

In the long term, 1, 3 and 5 year survival rates for all patients were 85.5, 83.7 and $80.7 \%$ respectively. The 
survival rates in terms of procedural groups, there was no significant difference among the groups $(\mathrm{P}=0.71$, Fig. 1 ).

\section{Discussion}

We investigated hemodynamic performance of double valve prostheses and effects of long term outcomes of functional TR following DVR. We found no significant difference among the groups according to potential complications and in-hospital mortality, so DVR can be safely due to a low operative mortality irrespectively of tricuspid valve intervention. However, the degree of postoperative TR varies according to whether to perform tricuspid intervention. The degree of TR decreased significantly in both the ring annuloplasty and the TDVA group, the rates of moderate and severe TR were significantly lower in the ring annuloplasty group than others. As for the survival rates there was no significant difference among the groups. This study found that female gender was an independent marker for the progression of TR. Also female patients have more rheumatic valvular pathologies than male patients.

DVR with tricuspid valve surgery is related to a high mortality risk ranging from $10 \%$ and $50 \%$ (14). DVR has better outcomes thanks to improvement in the technical approach of valve operations (15). Recently, in-hospital mortality has been low regardless of concomitant tricuspid valve intervention (15-17). In this study, the rate of in-hospital mortality was the same as that of current studies (\%11 in all groups with or without tricuspid intervention). The condition of tricuspid valve has been reported to be a significant marker of mortality pertaining to valve in patients undergoing DVR (18-21). In this study, although in-hospital mortality did not significantly differ among the three groups, the degree of postoperatively TR was observed to be significantly different considering tricuspid intervention.

The presence of mitral valve prostheses in patients undergoing DVR has been put forward to have a negative influence on the hemodynamic performance of the prosthetic aortic valve, since the movement impedes of intervalvular fibrous structure during systole. Additionally if the mitral valve prosthesis is incorrectly implanted, the mitral valve may interfere with the movement of the intervalvular fibrous body during systole or may cause narrowing of the left ventricular outflow tract (LVOT) (2). Lee and colleagues that aortic patient-prosthesis mismatch in DVR, no matter what size of mitral prosthesis was used, resulted in elevated TR, however this did not significantly correlated with all survival rates (22). Jeong et all. found that the postoperative aortic trans-prosthetic mean pressure gradient (TMPG) was $13.5 \mathrm{~mm} \mathrm{Hg}$ for the not only mechanical but also bio-prostheses, being the same in isolated aortic valve replacements(14). As with the findings of the presence study, the aortic TMPG was 13.3 for both mechanical and bio-prostheses and we did not found any differences in the development of TR according to TMPG, which was not different in either the bio-prosthesis or the mechanical valve.

Markers of late TR after left sided valve surgery, including AF, left atrial dilatation, and impaired right ventricular function has been reported $(23,24)$. Tricuspid valve interventions that are carried out simultaneously with left-sided valve surgery have been suggested in patients with severe TR, however, similar interventions in patients with less than moderate TR is challenging (25). In our study, the No-TAP group had significantly lower levels of maximum mitral gradient, PAP and degree of TR compared with the other two groups. We observed that the possible reason for elevated TR may be related to the TDVA group with large LAD before the surgery and there was no significant regression on LAD after the surgery. Song et al. delineated that female gender was an independent marker of elevated TR, which was likely assigned to the impact of a comparatively increased frequency of rheumatic involvement in the mitral valve, such as our findings (3). Jeong et all. found that female gender was correlated with late increased TR(14). As in our study, female gender and preoperative degree of TR were independent markers for the progression of TR. Additionally there were no significant differences according to BMI, degree of preoperative TR and tricuspid annulus diameter between the male and female patients.

Lee et all. despite the high proportion of patients (83\%) with rheumatic valvular pathology, this factor showed no association with late significant TR progression in univariate analysis (22) Such as our findings.

Recently, support the strategy of correcting mild-to-moderate functional TR at the time of MV replacement 
to maintain TV function and improve clinical outcomes (26). In a retrospective study involving 638 patients who had less than moderate functional TR undergoing left-side heart surgery without TV surgery, late significant TR was detected in $7.7 \%$ of patients during a mean follow-up period of 64 months(4). In another study on the impact of TV repair for functional TR in patients undergoing MV replacement, concomitant TV repair prevented TR progression and chronic heart failure (CHF) in patients (26). Of note, Kim and colleagues did not show differences in survival or incidence of CHF between the repair and non-repair groups. The discrepancies in the influence of TV repair on survival or CHF between this study and the cited studies are probably attributable to different study populations. Inclusion of patients with severe TR may have magnified the effect of TV repair on clinical outcomes in the cited studies (25). Presently, in the long period, 1, 3 and 5-years survival rates for all patients were 85.5(no -TAP), 83.7(Ring Ann) and 80.7\% (TDVA) respectively. ). In particular, CPB duration was significantly longer in ring annuloplasty group. Looking at the postoperative outcomes, there was no significance difference among the groups with regard to potential complications and in-hospital mortality.

Ring annuloplasty has been reported to be better than suture annuloplasty for the repair of $\operatorname{TR}(6)$. Kim et al. found that both techniques showed similar efficacy in restoring and maintaining TV function(25). In our study, the rates of moderate and severe TR were significantly lower in ring annuloplasty group. Looking at the survival rates in terms of procedural groups, there was no significant difference among the groups. A durable mitral valve repair and preservation of sinus rhythm are the keys to preventing late TR progression(27). The present study, the only parameter of preoperative AF was significantly lower in no-TAP group.

2017 ESC/EACTS guidelines recommended; Surgery should be considered in patients with mild or moderate secondary tricuspid regurgitation with a dilated annulus $\left(>\_40 \mathrm{~mm}\right.$ or $>21 \mathrm{~mm} / \mathrm{m} 2$ by $2 \mathrm{D}$ echocardiography) undergoing left-sided valve surgery (class 2a). Surgery may be considered in patients undergoing left-sided valve surgery with mild or moderate secondary tricuspid regurgitation even in the absence of annular dilatation when previous recent right-heart failure has been documented (class 2b). (28). Despite, we performed tricuspid intervention in patients with moderate and severe TR or dilated annulus $\left(>_{-} 40 \mathrm{~mm}\right)$; the degree of TR decreased significantly both ring annuloplasty and TDVA group and no-TAP group was associated with severe TR on long term follow up. In addition degree of preoperative TR was independent marker for the progression of long term TR.

\section{Conclusion}

Although the degree of postoperative TR varies according to whether to perform a tricuspid intervention, double valve replacement can be safe due to low operative mortality independently from tricuspid valve intervention. The severity of TR diminished significantly in both the ring annuloplasty and the TDVA group, but the ratio of moderate and severe TR were significantly lower in the ring annuloplasty group. In contrast to widely adopted, tricuspid intervention should be considered (especially ring annuloplasty) in female patients who have mild to moderate TR and $<40 \mathrm{~mm}$ annular diameter. Since our study has a retrospective design and included observational data from a single-center with a relatively small cohort, further prospective and multicenter studies are needed.

\section{References}

1. Spiliotopoulos K, Armstrong S, Maganti M, David TE. Does the mitral valve prosthesis adversely affect the hemodynamic performance of the aortic valve prosthesis in patients with double valve replacement? J Thorac Cardiovasc Surg 2012;143:S74eS77.

2. Jeong DS, Park PW, Mwambu TP, Sung K, Kim WS, Lee YT, Park SJ, Park SW. Tricuspid reoperation after left-sided rheumatic valve operations. Ann Thorac Surg 2013;95:2007e2013.

3. Song H, Kim MJ, Chung CH, Choo SJ, Song MG, Song JM, Kang DH, Lee JW, Song JK. Factors associated with development of late significant tricuspid regurgitation after successful left-sided valve surgery. Heart 2009;95:931e936

4. Nath J, Foster E, Heidenreich PA. Impact of tricuspid regurgitation on long-term survival. J Am Coll Cardiol 2004;43:405e9. 
5. Tang GHL, David TE, Singh SK, Singh, S. K., Maganti, M. D., Armstrong, S. et. al., Tricuspid valve repair with an annuloplasty ring results in improved long-term outcomes. Circulation. 2006;114:577I581.

6. McCarthy PM, Bhudia SK, Rajeswaran J, Hoercher, K. J., Lytle, B. W., Cosgrove, D. M. et al., Tricuspid valve repair: durability and risk factors for failure. J Thorac Cardiovasc Surg. 2004;127:674685.

7. Navia JL, Nowicki ER, Blackstone EH, Blackstone, E. H., Brozzi, N. A., Nento, D. E., et al. Surgical management of secondary tricuspid valve regurgitation: annulus, commissure, or leaflet procedure? J Thorac Cardiovasc Surg. 2010;139:1473-1482.

8. Hermans H, Tjahjono M, Faes D, Belmans A, Meuris B, Herijgers P, et. al. Mid-term follow up of triple valve surgery in a western community: predictors of survival. J Heart Valve Dis 2010;19:644e651; discussion 652.

9. Groves PH, Lewis NP, Ikram S, Maire R, Hall RJ. Reduced exercise capacity in patients with tricuspid regurgitation after successful mitral valve replacement for rheumatic mitral valve disease. Br Heart J 1991;66:295-301.

10. Koelling TM, Aaronson KD, Cody RJ, Bach DS, Armstrong WF. Prognostic significance of mitral regurgitation and tricuspid regur- gitation in patients with left ventricular systolic dysfunction. Am Heart J 2002;144:524-9.

11. King RM, Schaff HV, Danielson GK, Gersh BJ, Orszulak TA, Piehler JM, et al. Surgery for tricuspid regurgitation late after mitral valve replacement. Circulation 1984;70(3 Pt 2):I193-7.

12. Czer LS, Maurer G, Bolger A, DeRobertis M, Kleinman J, Gray RJ, et al. Tricuspid valve repair. Operative and follow-up evaluation by Doppler color flow mapping. J Thorac Cardiovasc Surg 1989;98: 101-10.

13. Goldman ME, Guarino T, Fuster V, Mindich B. The necessity for tricuspid valve repair can be determined intraoperatively by two- dimensional echocardiography. J Thorac Cardiovasc Surg 1987;94: 542-50.

14. Jeong, D. S., Park, P. W., Sung, K., Kim, W. S.,Lee, Y. T. Determinants of late tricuspid regurgitation after aortic-mitral double valve replacement. The American journal of cardiology, (2017). 119(10), 1643-1649.

15. Suri RM, Thourani VH, Englum BR, Rankin JS, Badhwar V, Svensson LG, et. al., The expanding role of mitral valve repair in triple valve operations: contemporary North American outcomes in 8,021 patients. Ann Thorac Surg 2014;97:1513e1519; discussion 1519.

16. Vohra HA, Whistance RN, Hechadi J, de Kerchove L, Fuller H, Noirhomme P, et. al., Long-term outcomes of concomitant aortic and mitral valve repair. J Thorac Cardiovasc Surg 2014;148: 454e460.

17. Vassileva CM, Li S, Thourani VH, Suri RM, Williams ML, Lee R, Rankin JS. Outcome characteristics of multiple-valve surgery: com- parison with single-valve procedures. Innovations (Phila) 2014;9: 27e32.

18. Arom KV, Nicoloff DM, Kersten TE, Northrup WF 3rd, Lindsay WG, Emery RW. Ten-year follow-up study of patients who had double valve replacement with the St. Jude Medical prosthesis. J Thorac Cardiovasc Surg 1989;98:1008e1015; discussion 1015e1016.

19. Kratz JM, Crawford FA Jr, Sade RM, Crumbley AJ, Stroud MR. St. Jude prosthesis for aortic and mitral valve replacement: a ten-year experience. Ann Thorac Surg 1993;56:462e468.

20. John S, Ravikumar E, John CN, Bashi VV. 25-year experi- ence with 456 combined mitral and aortic valve replacement for rheumatic heart disease. Ann Thorac Surg 2000;69: 1167e1172.

21. Wang G, Sun Z, Xia J, Deng Y, Chen J, Su G, Ke Y. Predictors of secondary tricuspid regurgitation after left-sided valve replacement. Surg Today 2008;38:778e783.

22. Lee, S. H., Youn, Y. N., Chang, B. C., Joo, H. C., Lee, S.,Yoo, K. J. Et all. Effect of patient-prosthesis mismatch in aortic position on late-onset tricuspid regurgitation and clinical outcomes after double valve replacement. Yonsei medical journal,2017, 58(5), 968-974.

23. Matsuyama K, Matsumoto M, Sugita T, Nishizawa J, Tokuda Y, Matsuo T. Predictors of residual tricuspid regurgitation after mitral valve surgery. Ann Thorac Surg 2003;75:1826e1828.

24. Vahanian A, Alfieri O, Andreotti F, Antunes MJ, Baron-Esquivias G, Baumgartner H, et. al. ESC 
Committee for Practice Guidelines (CPG), Joint Task Force on the Management of Valvular Heart Disease of the European Society of Cardiology, European Association for Cardio-Thoracic Surgery. Guidelines on the manage- ment of valvular heart disease (version 2012): the Joint Task Force on the Management of Valvular Heart Disease of the European Society of Cardiology (ESC) and the European Association for Cardio-Thoracic Surgery (EACTS). Eur J Cardiothorac Surg 2012;42:S1eS44.

25. Kim, J. B., Yoo, D. G., Kim, G. S., Song, H., Jung, S. H., et.al. Mild-to-moderate functional tricuspid regurgitation in patients undergoing valve replacement for rheumatic mitral disease: the influence of tricuspid valve repair on clinical and echocardiographic outcomes. Heart, 2012 . 98(1), 24-30.

26. Chan V, Burwash IG, Lam BK, Auyeung, T., Tran, A., Mesana, T. G., et al. Clinical and echocardiographic impact of functional tricuspid regurgitation repair at the time of mitral valve replacement. Ann Thorac Surg 2009;88:1209e15.

27. Ito, H., Mizumoto, T., Sawada, Y., Fujinaga, K., Tempaku, H., Shimpo, H., et. al. Determinants of recurrent tricuspid regurgitation following tricuspid valve annuloplasty during mitral valve surgery. Journal of cardiac surgery,2017; 32(4), 237-244.

28. Baumgartner, H., Falk, V., Bax, J. J., De Bonis, M., Hamm, C., Holm, P. J., et. al. 2017 ESC/EACTS guidelines for the management of valvular heart disease. European heart journal, 2017; 38(36), 27392791 .

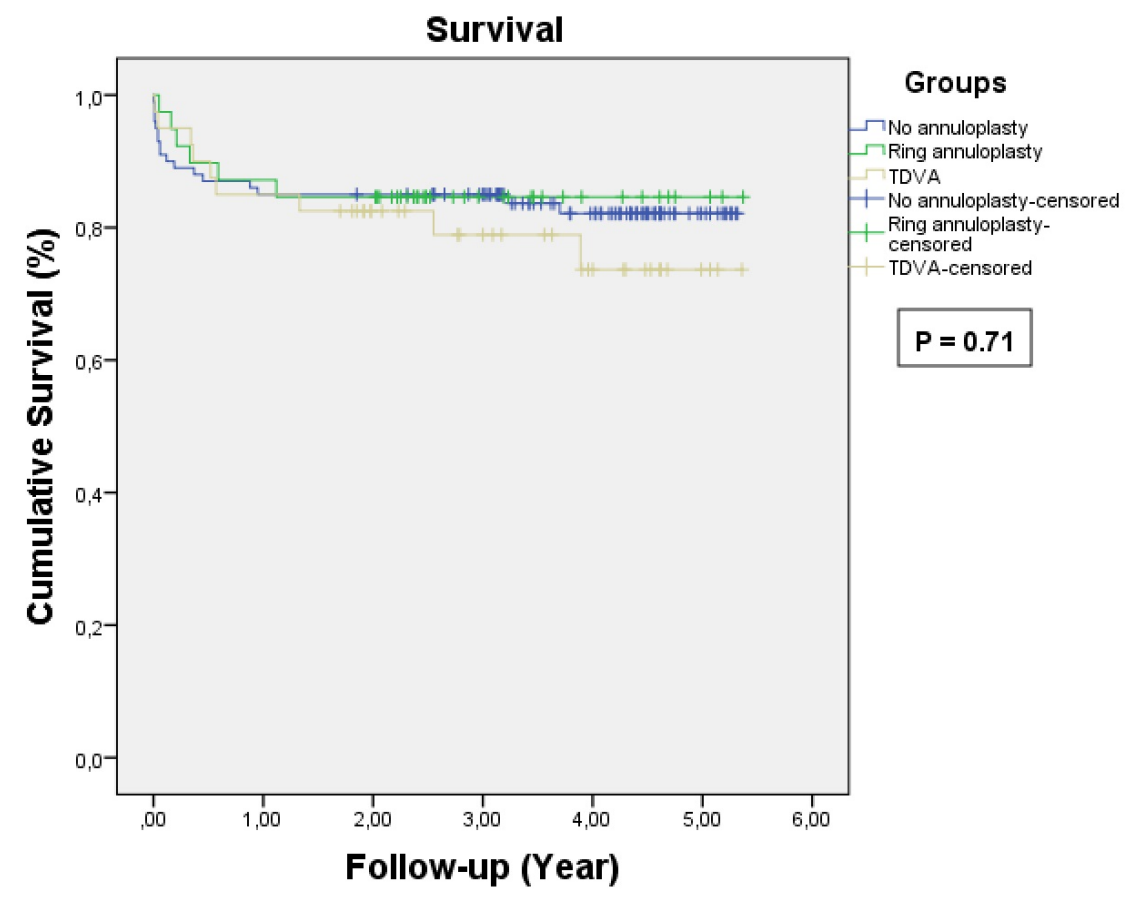

Figure 1: Survival rates

\section{Hosted file}

Table 1,2,3,4.docx available at https://authorea.com/users/322302/articles/451333-thefactors-affecting-long-term-tricuspid-regurgitation-after-double-valve-replacement 